\title{
Gaze Interaction in the Post-WIMP World
}

$\begin{array}{ll}\begin{array}{l}\text { Andreas Bulling } \\ \text { Computer Laboratory } \\ \text { University of Cambridge } \\ \text { Cambridge, UK } \\ \text { andreas.bulling@acm.org }\end{array} & \begin{array}{l}\text { Robert J. K. Jacob } \\ \text { Computer Science } \\ \text { Tufts University } \\ \text { Medford, MA 02155 USA } \\ \text { jacob@cs.tufts.edu }\end{array} \\ & \\ \text { Raimund Dachselt } & \text { Sophie Stellmach } \\ \text { Interactive Media Lab } & \text { Interactive Media Lab } \\ \text { Technische Universität Dresden } & \text { Technische Universität Dresden } \\ \text { Dresden, Germany } & \text { Dresden, Germany } \\ \text { dachselt@acm.org } & \text { stellmach@acm.org } \\ & \\ & \\ \text { Andrew T. Duchowski } & \text { Veronica Sundstedt } \\ \text { School of Computing } & \text { School of Computing } \\ \text { Clemson University } & \text { Blekinge Inst. of Technology } \\ \text { Clemson, SC 29634 USA } & \text { 37139 Karlskrona, Sweden } \\ \text { duchowski@acm.org } & \text { veronica.sundstedt@bth.se }\end{array}$

Copyright is held by the author/owner(s)

CHI 2013 Extended Abstracts, April 27-May 2, 2013, Paris, France.

ACM 978-1-4503-1952-2/13/04.

\begin{abstract}
This workshop is a follow-up to our successful Special Interest Group (SIG) meeting at CHI '12. The SIG focused on eye gaze as a highly effective, seamless, and fast means of human-computer interaction in various contexts, but showed that suitable applications and overall convenience are still lacking. Consequently, we see an expressive need for continued in-depth discussion on how the diversity of application contexts could specifically benefit from the incorporation of the user's gaze. In this regard, we concentrate on gaze interaction in combination with post-WIMP interaction styles as opposed to traditional desktop setups to broaden the view for novel ways to interact with eye gaze. The objective of the $\mathrm{CHI}$ '13 workshop is to further promote this emerging field by addressing fundamental research questions regarding novel gaze-based interaction in the post-WIMP world.
\end{abstract}

\section{Keywords}

Eye tracking; gaze; multimodal; interaction; post-WIMP

\section{ACM Classification Keywords}

H.5.2 [Information Interfaces and Presentation]: User Interfacesinput devices and strategies.

\section{General Terms}

Design, Human Factors 


\section{Introduction}

A diversity of post-WIMP interaction styles has emerged in recent years [5]. These interaction styles are designed for a variety of novel input and display technologies becoming pervasive in everyday life. While significant progress has been made in fields such as multi-touch, gestural, tangible, and 3D interaction, eye gaze has not yet been sufficiently explored for convenient and seamless integration. However, there is great potential for incorporating eye gaze as a supporting modality for more natural interaction. Examples include attentionaware interface adaptations [10], the combination of gaze with hand gestures or mobile devices for seamless navigation in complex information spaces [6, 13], and selection of distant targets on large-sized screens or multi-touch tables via gaze $[7,9,12]$. Thus, the development of such natural interaction techniques integrating gaze with other post-WIMP modalities such as multi-touch, hand gestures, speech, or tangibles is a promising avenue of research [3]

Novel ways of interaction are fueled by continuous advancement of eye tracking technology offering new opportunities for incorporating eye gaze as a supporting input modality. However, while eye tracking technology is improving and the general course of research is clear (e.g., calibration-free and even mobile gaze tracking), a clear vision of gaze as an interaction modality is missing. To foster novel means of interaction, a number of issues need to be carefully dissected, including aspects of interaction design, technological development, evaluation issues as well as of privacy and of social implications. The goal of this workshop is to bring together researchers and practitioners who are concerned with the design, development, and implementation of new applications and services combining gaze with other input modalities.

\section{Related Venues and Workshop Objectives}

In recent years, the interest in gaze-based interaction has increased as more comfortable, affordable and precise tracking systems become available [1, 4]. This is also evidenced by an increasing amount of papers and projects discussing various concepts, interaction techniques, and experiments related to eye gaze as an input modality [2, 7, 8, 11, 13]. In addition, eye tracking-related conferences (e.g., ETRA and NGCA) and workshops (e.g., IWGSI, IUI, PETMEI, and DUET) have proliferated, indicating increased interest and growth of the research community. While these venues are all related to eye tracking, the topics are often specialized, focusing on improved tracking algorithms, human machine communication, pervasive eye tracking and mobile eye-based interaction, and gaze-controlled applications. The SIGGRAPH 2010 course on gaze input in games and Special Interest Groups (SIGs) at $\mathrm{CHI} 2011$ and $\mathrm{CHI} 2012$ suggest a need for discussing gaze as an input modality in a more general sense.

At our CHI '12 SIG we emphasized the need for more indepth discussions on gaze as a supplementary interaction modality in the post-WIMP world, including various application areas. However, the time slot of the SIG only allowed us to scratch the surface, identifying several interesting application examples, such as gaze-supported home controls, attentive car windshields, or gaze-controlled technical equipment in an operation room. There still remains a need for further discussions for how gaze input can be seamlessly and conveniently integrated in such contexts. More generally, what is needed is a better knowledge of the design of gaze-supported interaction, e.g., principles or guidelines that would facilitate and accelerate further development.

At the $\mathrm{CHI}$ '13 workshop we intend to address possibilities, limitations, and design considerations for gaze interaction in the post-WIMP world by providing a venue for building a re- 
search community around this evolving topic. Therefore, we aim to generate new ideas, to consolidate this field and to specify promising and still insufficiently investigated research directions, building on our successful introductory discussions from our SIG at $\mathrm{CHI} 12$.

\section{Workshop Research Questions}

One intent of this workshop is to raise interest in this emerging topic and bring together researchers and practitioners who are interested in the exploration and development of new applications and services using a combination of gaze and postWIMP interfaces. Several issues require in-depth discussion, including but not limited to the following:

- What examples of gaze interaction in the post-WIMP world already exist?

What went well and what went wrong? What were lessons learned?

\section{- How can post-WIMP interaction benefit from gaze?}

What can a user's visual attention indicate? How could particular user groups, tasks, and contexts benefit?

What new application areas for gaze-supported interaction can emerge? How could gaze input facilitate interaction with diverse output devices? How can integration of gaze data with other modalities be made seamless?

- What needs to be considered for the design of (multimodal) post-WIMP gaze interaction?

What are general design guidelines, methods, and models that have to be taken into account for gazesupported interaction? What are particular challenges and how could they be tackled?

The main goal of this workshop is to lay the foundations for a community of researchers and practitioners interested in exploring novel ways to incorporate gaze input beyond the traditional desktop and WIMP metaphor. Our workshop offers a forum for exchanging experiences, bringing forward novel ideas, and forming new and long-lasting collaborations.

\section{Audience}

The workshop welcomes PhD students, senior researchers, as well as practitioners and industrial partners alike. We aim at creating a strong interdisciplinary research community linking various interest groups from different fields, such as interaction design, cognitive science, psychology, CSCW, and eye tracking. We hope the workshop will generate ideas that suggest further promising research directions to the $\mathrm{CHI}$ community.

\section{Organization}

We have already established a website ${ }^{1}$ on which updated information about the workshop will be made available (e.g., scope, call for papers, organizers, and organization).

We envision five main parts for our one-day workshop:

1. Introduction: A brief welcome of the attendees, introduction to the aim of this workshop and expectations of its attendees.

2. Paper presentations: Short talks about both position statements and workshop papers accepted to the workshop to lay the foundations for later brainstorming discussions.

3. Technology \& Research Demos: Before diving into group work and detailed discussions, we invite people from industry and research alike to provide insights into their current research and technology by giving some hands-on demonstrations of their work.

4. Envisioning the future: Smaller breakout groups will be formed to flesh out potential future gaze-based interaction scenarios and applications. The goal of this group

${ }^{1}$ See: http://www.gaze-interaction.net 
work including sketching and rapid prototyping methods is to come up with ideas, which altogether contribute to a broad vision on where gaze interaction could and should be heading in the short and long term. Among the key aspects to be considered in the group work are the following four:

(a) User contexts, e.g., public displays at airports, multi-display settings in meetings, multi-touch tables in museums, etc.;

(b) User groups, e.g., machine operators, experts vs. novices, accessibility issues, etc.;

(c) Interaction tasks, e.g., target acquisition, object manipulation, visual search, etc.;

(d) Multimodal combinations, e.g., gaze and hand gestures, speech, handhelds.

All projects of each group are pitched to the audience in a short presentation and shortly discussed.

5. Breakout Session for a research agenda: Building on the whole workshop experiences, a final brainstorming session will summarize important issues and research topics. Each participant is asked to contribute to collecting topics and components, which are then clustered to set up a broad research agenda in the field.

\section{References}

[1] Babcock, J. S., and Pelz, J. B. Building a lightweight eyetracking headgear. In Proc. of ETRA '04, ACM (New York, NY, USA, 2004), 109-114.

[2] Bardins, S., Poitschke, T., and Kohlbecher, S. Gazebased interaction in various environments. In Proc. VNBA '08 (2008), 47-54.

[3] Bulling, A., Dachselt, R., Duchowski, A., Jacob, R., Stellmach, S., and Sundstedt, V. Gaze interaction in the postwimp world. In Proc. of CHI EA '12, ACM (New York, NY, USA, 2012), 1221-1224.
[4] Bulling, A., and Gellersen, H. Toward Mobile Eye-Based Human-Computer Interaction. IEEE Pervasive Computing 9, 4 (2010), 8-12.

[5] Jacob, R., Girouard, A., Hirshfield, L., Horn, M., Shaer, O., Solovey, E., and Zigelbaum, J. Reality-based interaction: a framework for post-WIMP interfaces. In Proc. $\mathrm{CHI}$ '08 (2008), 201-210.

[6] Stellmach, S., and Dachselt, R. Investigating gazesupported multimodal pan and zoom. In Proc. of ETRA '12, ACM (New York, NY, USA, 2012), 357-360.

[7] Stellmach, S., and Dachselt, R. Look \& touch: Gazesupported target acquisition. In Proc. CHI'12 (2012).

[8] Stellmach, S., Stober, S., Nürnberger, A., and Dachselt, R. Designing gaze-supported multimodal interactions for the exploration of large image collections. In Proc. NGCA '11 (2011), 1-8.

[9] Turner, J., Bulling, A., and Gellersen, H. Combining gaze with manual interaction to extend physical reach. In Proc. of PETMEI '11, ACM (New York, NY, USA, 2011), 33-36.

[10] Vertegaal, R., Shell, J., Chen, D., and Mamuji, A. Designing for augmented attention: Towards a framework for attentive user interfaces. Computers in Human Behavior 22, 4 (July 2006), 771-789.

[11] Wilcox, T., Evans, M., Pearce, C., Pollard, N., and Sundstedt, V. Gaze and voice based game interaction: the revenge of the killer penguins. In SIGGRAPH Posters (2008), 81.

[12] Yamamoto, M., Komeda, M., Nagamatsu, T., and Watanabe, T. Development of eye-tracking tabletop interface for media art works. In Proc. of ITS'10, ACM (New York, NY, USA, 2010), 295-296.

[13] Yoo, B., Han, J.-J., Choi, C., Yi, K., Suh, S., Park, D., and Kim, C. 3D user interface combining gaze and hand gestures for large-scale display. In CHI EA '10 (2010), 3709-3714. 